\title{
Schistosomiasis of the Prostate in Sokoto, Nigeria: a case series
}

\author{
Abdullahi Khalid ${ }^{1 *} \mathbb{0}$, Abdullah Abdulwahab-Ahmed ${ }^{1}$, Ismaila Arzika Mungadi ${ }^{1}$, Kabiru Abdullahi², \\ Ngwobia Peter Agwu' and Abubakar Sadiq Muhammad'
}

\begin{abstract}
Background: Prostate schistosomiasis is a chronic inflammatory condition of the prostate. In an endemic environment, it may mimic the sinister condition of the prostate in its clinical presentation. Despite the high prevalence of urinary schistosomiasis documented in our environment, prostate involvement is rarely encountered in routine clinical practice. We report and share our experience in the management of two cases of prostate schistosomiasis that presented with abnormal digital rectal examination and elevated prostate-specific antigen suggestive of prostate cancer but proved otherwise by the histology report.

Case presentation: The first patient was a 50-year-old man who presented with a history of voiding and storage lower urinary tract symptoms (LUTS) and urinary retention relieved by urethral catheterization. There was no other significant history except for past childhood terminal hematuria. The remarkable findings on physical examination were in the digital rectal examination which revealed enlarged prostate, hard, nodular, obliterated median groove, and restricted mobility of overlying rectal mucosa. The total serum prostate-specific antigen (PSA) was $1.2 \mathrm{ng} / \mathrm{ml}$. Transrectal ultrasound-guided prostate biopsy and histology confirmed prostate schistosomiasis on a background of nodular hyperplasia. The second patient was an 80-year-old man who presented with a history of voiding and storage lower urinary tract symptoms (LUTS) and urinary retention relieved by urethral catheterization. There was no other significant history and no past childhood terminal hematuria. There were no remarkable findings on physical examination except for the enlarged prostate with benign findings on digital rectal examination. The initial total serum prostate-specific antigen (PSA) was $31.0 \mathrm{ng} / \mathrm{ml}$. The repeated PSA was $7.5 \mathrm{ng} / \mathrm{ml}$ after antimicrobial therapy. Transrectal ultrasound-guided prostate biopsy and histology confirmed prostate schistosomiasis on a background of nodular hyperplasia. He had two sessions of Bipolar Transurethral Resection of the Prostate following failed medical treatment.
\end{abstract}

Conclusions: Schistosomiasis of the prostate may mimic the clinical or biochemical presentation of prostate cancer. The attending physician requires high index of suspicion to arrive at this diagnosis, especially for the urologist practicing in an endemic area.

Keywords: Prostate, Schistosomiasis, Presentation, Transurethral resection of prostate, Nigeria

*Correspondence: kalabduka@gmail.com

1 Tetfund Centre of Excellence in Urology and Nephrology, Institute of Urology and Nephrology, Usmanu Danfodiyo University and Usmanu Danfodiyo University Teaching Hospital, Sokoto, Nigeria

Full list of author information is available at the end of the article

\section{Background}

Schistosomiasis is a commonly encountered condition in Africa particularly sub-Saharan Africa. It is an endemic disease and well-known neglected tropical disease ranking second after Hookworm infestation in this region (Hotez and Kamath 2009). It affects about twenty-five percent of people which is equal to the 192 million population of sub-Saharan Africa (Hotez and Kamath 2009). Nigeria which is a part of sub-Saharan Africa is not 
spared of this disease and its sequelae as several reports documented its involvement of gastrointestinal and urogenital systems (Singh et al. 2016; Uwaezuoke et al. 2007; Uko et al. 1993; Pukuma and Musa 2007; Mafiana and Adesanya 1999). Its prevalence varies from region to region in Nigeria. In the North-western part, overall prevalence of urinary schistosomiasis as high as $60.8 \%$ has been documented in a study (Singh et al. 2016). It is a chronic inflammatory condition and clinical syndrome caused by digenetic trematodes of the genus Schistosoma with sequelae seen in multiple organ systems. The culprit systems that bear the greater brunt typically in this infestation are urogenital and gastrointestinal (Singh et al. 2016). Several schistosoma species have been identified but the urogenital and gastrointestinal diseases are known to be caused by Schistosoma hematobium and Schistosoma mansoni respectively.

The prostate is one of the urogenital organs that can be affected in schistosomiasis, though reported rarely when compared to the affectation of bladder, ureter, and seminal vesicles. Even in schistosoma endemic environments, the picture of prostate schistosomiasis has not been documented to be a frequent occurrence. The urogenital organs often and typically involved in Schistosoma hematobium infestation include the bladder, ureters, and seminal vesicles (Barsoum 2013). It is paramount to note that prostate and vas deferens affectation occur less frequently (Barsoum 2013). In persons with urinary schistosomiasis, cell-mediated and immune-complex-mediated immune responses are observed. The cell-mediated immune responses to the presence of Schistosoma hematobium infection consequently lead to granulomata formation in the urinary tract (Barsoum 2013). The chronic inflammatory process and healing by fibrosis thus give rise to stricture and calcification in the urinary system. Other sequelae of this pathological process include secondary stone formation and bladder cancer as reported in some studies (Mungadi and Malami 2007).

Regardless of the urogenital organs involved, urinary schistosomiasis manifests with two clinicopathological forms namely inflammatory and fibrotic types (Barsoum 2013). The inflammatory phase occurs early and is characterized by cellular activity and the release of cytokines. The fibrotic type occurs late in the disease course. The effects of prostate fibrosis include less compliant organ and attendant bladder neck fibrosis leading to bladder outlet obstruction (BOO) (Bushman and Jerde 2016). Also fibrotic prostate may clinically feel hard and in addition, the recurrent prostate inflammation may influence the rise in prostate-specific antigen (PSA), an important and useful diagnostic armamentarium of prostatic disease patient evaluation. Therefore, these create the wrong impression of sinister conditions of the prostate such as prostate cancer at presentation. These clinicopathological and biochemical alterations caused by the prostate schistosomiasis, as well as prevalent lower urinary tract symptoms (LUTS), due to benign prostate enlargement (BPE) seen in middle-aged men can present as diagnostic and therapeutic challenges to the attending urologist who manages these patients.

Sokoto is the capital city of Sokoto State in North-western, Nigeria. It is endemic for schistosomiasis and located on the latitude $12^{\circ} 15^{\prime} 29^{\prime \prime}$ North of the equator and longitude $13^{\circ} 58^{\prime} 22^{\prime \prime}$ East of the Greenwich meridian (Auwal and Abdussalam 2015). It is located at an elevation of $296 \mathrm{~m}$ above sea level (Auwal and Abdussalam 2015). The state and her neighboring states endowed with rivers, dams, and irrigation farming systems serve as a breeding ground for the vectors of this disease (Adams 1991). The people of these states share common geographic and socio-economic characteristics. In addition, the predominant occupation of the inhabitants of this location being farming and fishing serves as perpetuating factors. These economic activities may explain partly, the inability to completely eradicate the disease.

Because of these, reports of schistosomiasis involving various organ systems are not uncommon in this environment. There are studies on some of the coexistence of schistosomiasis with other pathological conditions as well as sequelae of urinary schistosomiasis such as bladder cancer, post-schistosomal ureteral fibrosis, and upper tract urinary stones (Mungadi and Malami 2007; Aminu et al. 2014; Mungadi and Khalid 2021). Despite these, there have not been documented reports of prostate schistosomiasis. Hence, there is a need to report these cases to add to the body of knowledge and share our experience in these patients' management.

\section{Case presentation Case 1}

A 50-year-old man presented with voiding and storage lower urinary tract symptoms of year duration. The symptoms progressively worsened despite the use of medical therapy for benign prostate enlargement (BPE) culminating in acute urinary retention relieved by urethral catheterization. He had a past childhood history of terminal hematuria. There was no history of urethritis, urethral instrumentation, perineal or pelvic trauma. There were no constitutional symptoms of malignancy, family history of prostate cancer, or a suggestion of its metastasis.

Physical examination was not remarkable except the digital rectal examination that revealed an enlarged prostate, hard and nodular, obliterated median groove, and restricted mobility of overlying rectal mucosa. Abdominal ultrasound scan showed irregular mixed echogenic 
prostate. The prostate-specific antigen (PSA) was $1.2 \mathrm{ng} /$ $\mathrm{ml}$. He had a transrectal ultrasound-guided scan and prostate biopsy.

The histology report confirmed prostate schistosomiasis on a background of nodular hyperplasia as shown in Fig. 1a and b. This patient was lost to follow-up before the planned definitive treatment probably due to financial constraints.

\section{Case 2}

An 80-year-old man presented with voiding and storage lower urinary tract symptoms of nine months duration. The symptoms progressively worsened despite the use of medical therapy for benign prostate enlargement (BPE) culminating in acute urinary retention relieved by urethral catheterization. He had no past childhood history of terminal hematuria. There was no history of urethritis, urethral instrumentation, perineal or pelvic trauma. There were no constitutional symptoms of malignancy or family history of prostate cancer or features suggestive of prostate cancer metastasis.

Physical examination was not remarkable except for digital rectal examination that revealed an enlarged prostate, firm, preserved median groove, and mobile overlying rectal mucosa. Abdominal ultrasound scan showed enlarged prostate, with uniform parenchyma echogenicity and intact capsule. The prostate-specific antigen (PSA) was $31.0 \mathrm{ng} / \mathrm{ml}$. The repeated PSA was $7.5 \mathrm{ng} / \mathrm{ml}$ after antimicrobial treatment. He had a transrectal ultrasound scan and prostate biopsy.

The histology report confirmed prostate schistosomiasis on a background of nodular hyperplasia as shown

A

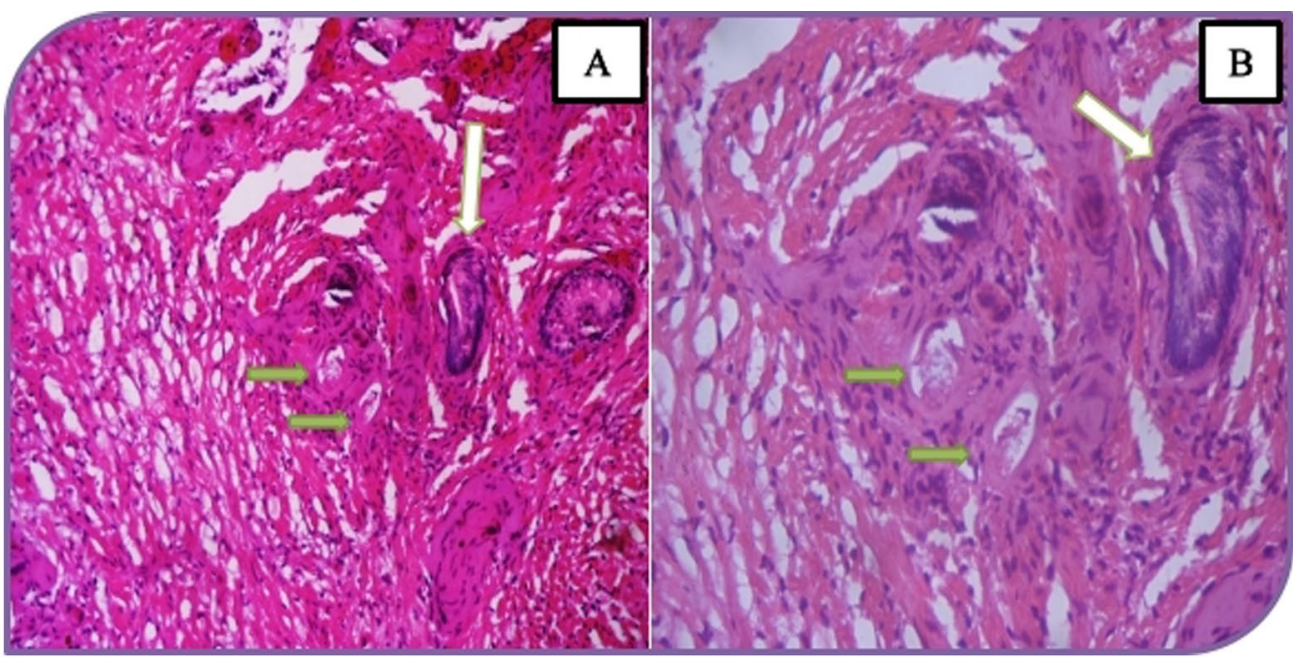

B

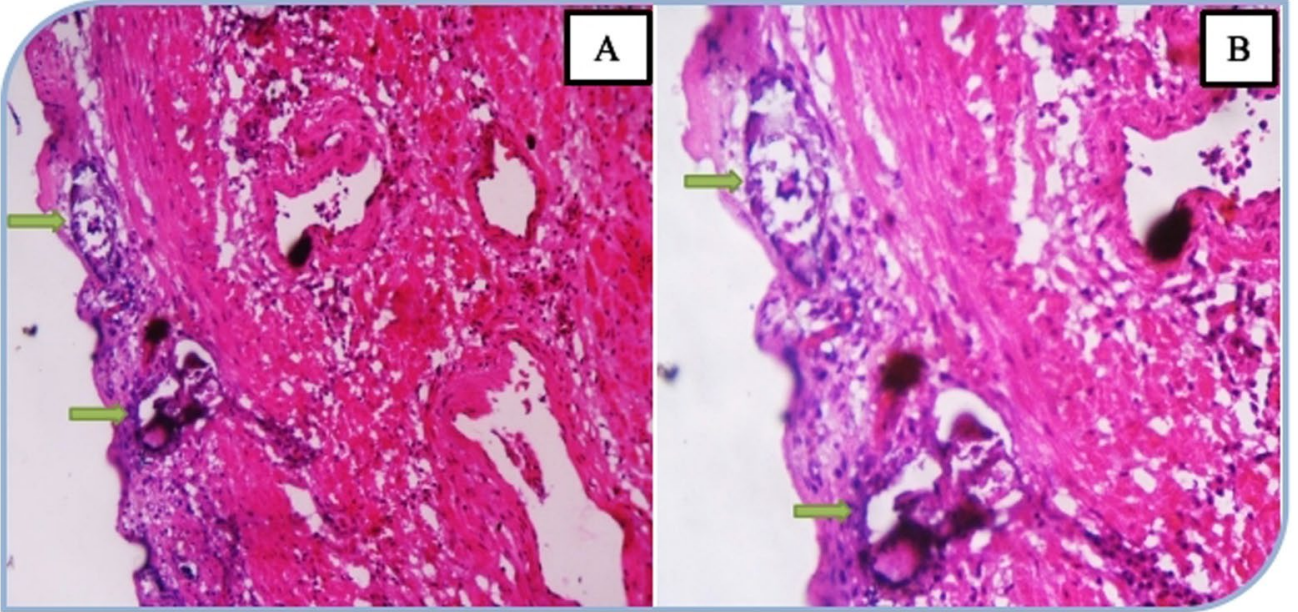

Fig. 1 a Nodular hyperplasia of the prostate with Schistosoma ova (green arrow) in myofibrous stroma. The white arrow is a benign gland. a $H$ \& $E$ X 200 while $\mathbf{b} \mathrm{H} \& \mathrm{E}$ X 400. b Nodular hyperplasia of the prostate with schistosoma ova (green arrow) in myofibrous stroma. $\mathbf{a}$. $\mathrm{H} \& \mathrm{E} X 200$ while $\mathbf{b} \mathrm{H}$ $\& E X 400$ 
in Fig. 2. He had two sessions of Bipolar Transurethral resection of the prostate following failed medical treatment. His symptoms resolved after surgical intervention with satisfactory follow-up visits.

\section{Discussion}

Lower urinary tract symptoms are the common reason for presentation to the urologist of men from their middle ages and beyond. This is common as a result of prostatic diseases such as benign prostate enlargement or prostate cancer. Other conditions that could cause LUTS in this age group include, but less often, urethral stricture, lumbar spondylosis, and neurogenic bladder. Prostate schistosomiasis is often not considered as a possible differential diagnosis of LUTS or even BOO due to its rarity. Thus, it underscores the need for a high index of suspicion. In the two index cases, this was not considered until the histology finding confirmed the diagnosis. The ages of the index cases at presentation are well in keeping with the time of manifestations of LUTS as seen in the patients. However, to determine what the concurrent presence of $\mathrm{BPE}$ and prostate schistosomiasis contribute to LUTS and its severity may be difficult. In any case, LUTS in these patients may be due to BPH or prostate schistosomiasis, or both. Prostate schistosomiasis will better explain the severity of LUTS in the younger patient. This can also be a subject of debate. But it is worthy of note that patients who had childhood exposure to acute urinary schistosomiasis may be presenting in adulthood with sequelae of the chronic or fibrotic type which occur late in the disease progression (Barsoum 2013). The effects of prostate fibrosis with involvement of bladder neck will cause bladder neck stenosis and subsequent
BOO. This pathologic process can also explain the LUTS in the patients (Bushman and Jerde 2016).

In addition, this pathology may even be the underlying reason why benign prostatic hyperplasia $(\mathrm{BPH})$ patients who ought to have benefited from medical therapy often fail as seen in the index patients. Fibrotic prostate is a recognized cause of failed medical therapy. Failed medical therapy as shown by the development of recurrent urinary retention was one of the reasons the patients were prepared for the bipolar transurethral resection of the prostate (TURP). This choice of treatment has the advantage of addressing the static component of the $\mathrm{BPH}$, at the same time widening the bladder neck, thus minimizing the LUTS due to bladder neck stenosis from prostate schistosomiasis. One can argue that the known treatment options of bladder neck stenosis depend on whether the stenosis is a primary or secondary type. The common treatment options include urethral dilation, endoscopic bladder neck incisions, stenting, or abdominoperineal reconstruction of the bladder neck (Nicholson et al. 2017). These treatments are suitable for isolated causes of bladder neck stenosis. In combined bladder neck stenosis with $\mathrm{BPH}$ as may be the case here, we considered bipolar TURP a suitable option in their management due to the resolution of LUTS during the follow-up visit. Although one of the index cases was lost to follow-up, he could have also benefited from bipolar transurethral resection of the prostate, since he also had urinary retention due to failed medical therapy for his lower urinary tract symptoms. The reason for the loss to follow-up in the index case was not clear. However, financial constraints to the planned surgery may be likely because many patients still rely on out-of-pocket spending for their treatment.

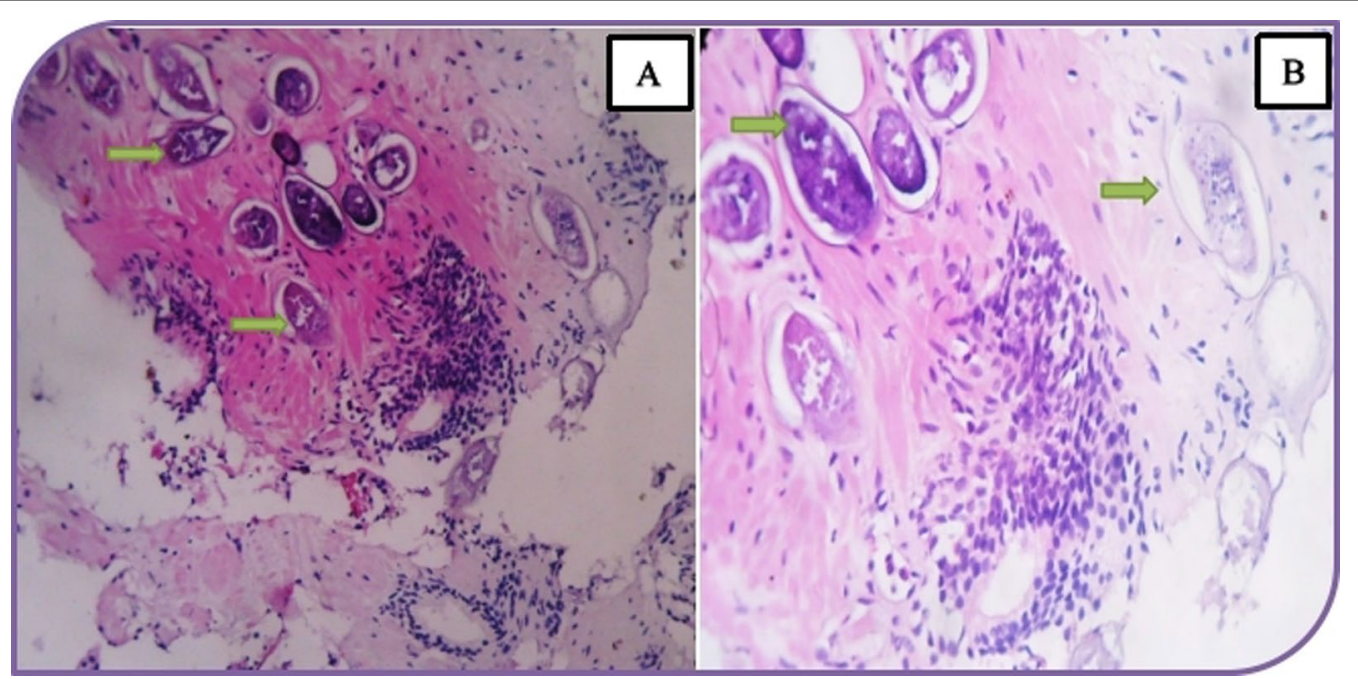

Fig. 2 Nodular hyperplasia of the prostate with Schistosoma ova (green arrow) in myofibrous stroma. a. H \& EX 200 while b H \& EX 400 
Also, the fibrotic prostate may clinically feel hard. The digital rectal examination in one of the patients revealed enlarged, hard, and nodular prostate with obliterated median groove as well as restricted mobility of overlying rectal mucosa. Although this patient's PSA was $1.2 \mathrm{ng} /$ $\mathrm{ml}$, we had a high index of suspicion of prostate cancer due to abnormal DRE until the histology result proved otherwise. Abnormal DRE and normal or low serum level of PSA can also be seen in patients on medical treatment with $5 \alpha$ reductase inhibitors such as dutasteride for $\mathrm{BPH}$ and patients with neuroendocrine prostate cancers. The recurrent prostate inflammation from schistosomal induced prostatitis can cause elevation of PSA as seen in one of the patients. This could explain the drop in the PSA from the initial value of $31.0 \mathrm{ng} / \mathrm{ml}$ to $7.5 \mathrm{ng} / \mathrm{ml}$ after antibiotic treatment observed in one of the patients. The elevated PSA which is a useful diagnostic tool of prostatic disease patient evaluation can pose a diagnostic challenge. This creates the wrong impression of a sinister condition of the prostate such as prostate cancer at presentation. It may unnecessarily expose patients to prostate biopsy with its attendant complications.

Prostate schistosomiasis can have a varied presentation. Since one of the index cases had clinical findings while the other biochemical finding suggestive of prostate cancer. Hence, prostate schistosomiasis is a diagnosis of exclusion. The significance of unrecognized prostate schistosomiasis is the attendant pathological changes caused in the prostate leading to bladder neck stenosis, BOO, and eventually obstructive nephropathy. We advocate for early decision and surgical treatment particularly minimally invasive like TURP, especially where facilities are available. The open surgical intervention in these patients may be fraught with difficulties due to background myofibrous prostate stroma as demonstrated in Figs. 1 and 2.

Sokoto is endemic for urinary schistosomiasis (Singh et al. 2016). It may explain the high burden of urological conditions associated with schistosomal endemicity here, such as upper tract calculi, lower ureteric obstruction post-schistosomal lower ureteral fibrosis, and bladder cancer of squamous histologic preponderance (Mungadi and Malami 2007; Aminu et al. 2014; Mungadi and Khalid 2021). Probably, a good number of these cases of prostate schistosomiasis may go undiagnosed since not all patients treated for LUTS due to BPE medically or at times surgically have the benefit of the histologic diagnosis. Also, in chronic stage schistosomiasis, Schistosoma ova may not be seen. Therefore, a high index of suspicion is required by the urologist practicing in a schistosomal endemic environment to exclude schistosomiasis of the prostate as a differential diagnosis of patients presenting with LUTS or BOO.

\section{Conclusions}

Schistosomiasis is a chronic inflammatory disease. Prostate involvement may present a diagnostic dilemma due to its varied clinical features and biochemical changes. Thus, it may mimic the clinical presentation of the dreadful disease of the prostate. Despite endemicity of schistosomiasis in our environment, prostate schistosomiasis may probably be rare or its diagnosis is often not suspected or missed during patient's evaluation for LUTS. Its coexistence with benign prostatic hyperplasia may compound the manifestation of LUTS in these patients leading to challenges in management. Therefore, a high index of suspicion as well as clinical and histopathological findings is required to arrive at this diagnosis, especially for the urologist practicing in an endemic area.

\section{Abbreviations}

LUTS: Lower urinary tract symptoms; PSA: Prostate-specific antigen; BOO: Bladder outlet obstruction; BPE: Benign prostate enlargement; TURP: Transurethral resection of prostate; $\mathrm{BPH}$ : Benign prostatic hyperplasia.

\section{Acknowledgements}

Not applicable.

\section{Authors' contributions}

All the authors are responsible for the design and implementation of the research. AK and AAA in collaboration with KA conceptualized this study and acquired the data. AK made the initial draft of this work. KA performed the histological examination, interpreted the prostate tissue specimen, and contributed to the revision of the initial draft. NPA and ASM did an additional literature search on the topic and contributed to writing the initial draft. AAA and IAM provided useful referenced materials, critically revised, and made a major contribution to the intellectual content of the initial manuscript. All authors read and approved the final manuscript.

\section{Funding}

None.

Availability of data and material

All data generated during this study are included in this published article.

\section{Declarations}

\section{Ethics approval and consent to participate}

Approval for the study "UDUTH/HREC/2016/438" was obtained from the Hospital Research and Ethics Committee (HREC) of Usmanu Danfodiyo University Teaching Hospital, Sokoto.

\section{Consent for publication}

Written informed consent was obtained from one of the patients who had an intervention for publication of this case report. The other patient was lost to follow-up before intervention as mentioned in the manuscript.

\section{Competing interests}

The authors declare that they have no competing interests.

\section{Author details}

${ }^{1}$ Tetfund Centre of Excellence in Urology and Nephrology, Institute of Urology and Nephrology, Usmanu Danfodiyo University and Usmanu Danfodiyo University Teaching Hospital, Sokoto, Nigeria. ${ }^{2}$ Department of Morbid Anatomy and Forensic Medicine, Usmanu Danfodiyo University and Usmanu Danfodiyo University Teaching Hospital, Sokoto, Nigeria. 
Received: 14 September 2021 Accepted: 3 February 2022

Published online: 16 February 2022

\section{References}

Adams WM (1991) Large scale irrigation in Northern Nigeria: performance and ideology. Trans Inst Br Geogr 16:287-300

Aminu MB, Abdullahi K, Dattijo LM (2014) Tubal ectopic gestation associated with genital schistosomiasis: a case report. Afr J Reprod Health 18:144-146

Auwal F, Abdussalam AF (2015) Changes in indices of daily temperature and precipitation extremes in Northwest Nigeria. Sci World J 10:18-26

Barsoum RS (2013) Urinary Schistosomiasis: Review. J Adv Res 4:453-459

Bushman WA, Jerde TJ (2016) The role of prostate inflammation and fibrosis in lower urinary tract symptoms. Am J Physiol Renal Physiol 311:817-821

Hotez PJ, Kamath A (2009) Neglected tropical diseases in sub-Saharan Africa: review of their prevalence, distribution, and disease burden. PLoS Negl Trop Dis 3:e412

Mafiana GF, Adesanya OO (1999) Urinary schistosomiasis in llewo - Orile, Ogun State. Nigeria Niger J Parasitol 15:32-34

Mungadi IA, Khalid A (2021) Spectrum of urological cases in a West African Tertiary Hospital. Ann Afr Med 20:14-18

Mungadi IA, Malami SA (2007) Urinary bladder cancer and schistosomiasis in North-Western Nigeria. West Afr J Med 26:226-229

Nicholson HL, Al-Hakeem Y, Maldonado JJ, Tse V (2017) Management of bladder neck stenosis and urethral stricture and stenosis following treatment for prostate cancer. Transl Androl Urol 6(Suppl 2):S92-S102

Pukuma MS, Musa SP (2007) Prevalence of urinary schistosomiasis among residents of Waduku in Lamurde Local Government Area of Adamawa State Nigeria. Niger J Parasitol 28:65-68

Singh K, Muddasiru D, Singh J (2016) Current status of schistosomiasis in Sokoto, Nigeria. Parasite Epidemiol Control 1:239-244

Uko IE, Adeoye GO, Tayo MM, Ogbe MG (1993) Evaluation of three diagnostic technique for urinary schistosomiasis (reagent strip, egg count and ELISA) in Kainji Lake Area Nigeria. Niger J Parasitol 14:65-73

Uwaezuoke JC, Anosike JC, Nwoke BEB, Dozie INS (2007) Urinary schistosomiasis in Ihitte Uboma Local Government Area of Imo State Nigeria. Niger J Parasitol 28:90-94

\section{Publisher's Note}

Springer Nature remains neutral with regard to jurisdictional claims in published maps and institutional affiliations.

\section{Submit your manuscript to a SpringerOpen ${ }^{\circ}$ journal and benefit from:}

- Convenient online submission

- Rigorous peer review

- Open access: articles freely available online

- High visibility within the field

- Retaining the copyright to your article 